\author{
Waghamore P. HaRina AND S. RAJEShWARI
}

\title{
NON-LINEAR DIFFERENTIAL POLYNOMIALS SHARING SMALL FUNCTION WITH FINITE WEIGHT
}

\begin{abstract}
The purpose of the paper is to study the uniqueness of entire and meromorphic functions sharing a small function with finite weight. The results of the paper improve and extend some recent results due to Abhijit Banerjee and Pulak Sahoo [3].

KEY WORDS: entire and meromorphic function, weighted sharing, nonlinear differential polynomials.
\end{abstract}

AMS Mathematics Subject Classification: 30D35.

\section{Introduction}

In this paper by meromorphic functions we will always mean meromorphic function in the complex plane.

Let $f$ and $g$ be two non-constant meromorphic functions and let $a$ be a finite complex number. We say that $f$ and $g$ share $a \mathrm{CM}$, provided that $f-a$ and $g-a$ have the same zeros with the same multiplicities. Similarly, we say that $f$ and $g$ share $a$ IM, provided that $f-a$ and $g-a$ have the same zeros ignoring multiplicities. In addition we say that $f$ and $g$ share $\infty$ $\mathrm{CM}$, if $\frac{1}{f}$ and $\frac{1}{g}$ share $0 \mathrm{CM}$, and we say that $f$ and $g$ share $\infty$ IM, if $\frac{1}{f}$ and $\frac{1}{g}$ share 0 IM.

We adopt the standard notations of value distribution theory (see [8]). We denote by $T(r)$ the maximum of $T(r, f)$ and $T(r, g)$. The notation $S(r)$ denotes any quantity satisfying $S(r)=o(T(r))$ as $r \rightarrow \infty$, outside of a possible exceptional set of finite linear measure.

Throughout this paper, we need the following definition.

$$
\Theta(a ; f)=1-\lim _{r \rightarrow \infty} \sup \frac{\bar{N}(r, a ; f)}{T(r, f)},
$$

where $a$ is a value in the extended complex plane. 
In 1959, Hayman [7] proved the following result.

Theorem A. Let $f$ be a transcendental entire function, and let $n(\geq 1)$ be an integer. Then $f^{n} f^{\prime}=1$ has infinitely many zeros.

In 2002, Fang and Fang [6] proved the following result.

Theorem B. Let $f$ and $g$ be two non-constant entire functions, and let $n(\geq 8)$ be an integer. If $f^{n}(f-1) f^{\prime}$ and $g^{n}(g-1) g^{\prime}$ share $1 C M$, then $f \equiv g$.

In the same year Fang [5] investigated the value sharing of more general non-linear differential polynomial than that was considered in Theorem B and obtained the following result.

Theorem C. Let $f$ and $g$ be two non-constant entire functions, and let $n$, $k$ be two positive integers with $n \geq 2 k+8$. If $\left[f^{n}(f-1)\right]^{(k)}$ and $\left[g^{n}(g-1)\right]^{(k)}$ share 1 CM then $f \equiv g$.

In 2004, Lin and Yi [14] considered the case of meromorphic function in Theorem B and obtained the following.

Theorem D. Let $f$ and $g$ be two non-constant meromorphic functions with $\Theta(\infty, f)>\frac{2}{n+1}$, and let $n(\geq 12)$ be an integer. If $f^{n}(f-1) f^{\prime}$ and $g^{n}(g-1) g^{\prime}$ share $1 C M$, then $f \equiv g$.

Natural inquisition would be to investigate the situation for meromorphic function in Theorem C. In this direction in 2008, Zhang [20] proved the following result.

Theorem E. Suppose that $f$ is a transcendental meromorphic function with finite number of poles, $g$ is a transcendental entire function, and let $n, k$ be two positive integers with $n \geq 2 k+6$. If $\left[f^{n}(f-1)\right]^{(k)}$ and $\left[g^{n}(g-1)\right]^{(k)}$ share $1 C M$, then $f \equiv g$.

To proceed further we require the following definition known as weighted sharing of values introduced by I. Lahiri [9] which measure how close a shared value is to being shared CM or to being shared IM.

Definition 1. Let $k$ be a non negative integer or infinity. For a $\in$ $\mathbb{C} \bigcup\{\infty\}$ we denote by $E_{k}(a ; f)$ the set of all a-points of $f$ where an a-point of multiplicity $m$ is counted $m$ times if $m \leq k$ and $k+1$ times if $m>k$. If $E_{k}(a ; f)=E_{k}(a ; g)$, we say that $f, g$ share the value a with weight $k$.

The definition implies that if $f, g$ share a value a with weight $k$, then $z_{0}$ is an a-point of $f$ with multiplicity $m(\leq k)$ if and only if it is an a-point of $g$ with multiplicity $m(\leq k)$ and $z_{0}$ is an a-point of $f$ with multiplicity $m(>k)$ if and only if it is an a-point of $g$ with multiplicity $n(>k)$, where $m$ is not necessarily equal to $n$.

We write $f, g$ share $(a, k)$ to mean that $f, g$ share the value a with weight $k$. Clearly if $f, g$ share $(a, k)$ then $f, g$ share $(a, p)$ for any integer 
$p, 0 \leq p<k$. Also we note that $f, g$ share a value a IM or CM if and only if $f, g$ share $(a, 0)$ or $(a, \infty)$ respectively.

In 2009, using the notion of weighted sharing of values, $\mathrm{Xu}$, Yi and Cao [15] proved the following result.

Theorem $\mathbf{F}$. Let $f$ and $g$ be two non-constant meromorphic functions, and $n(\geq 1), k(\geq 1)$ and $l(\geq 0)$ be three integers such that $\Theta(\infty, f)+$ $\Theta(\infty, g)>\frac{4}{n}$. Suppose $\left[f^{n}(f-1)\right]^{(k)}$ and $\left[g^{n}(g-1)\right]^{(k)}$ share $(1, l)$. If $l \geq 2$ and $n>5 k+11$ or if $l=1$ and $n>7 k+\frac{23}{2}$, then $f \equiv g$.

Recently, Li [13] proved the following result which rectify and at the same time improve Theorem F.

Theorem G. Let $f$ and $g$ be two non-constant meromorphic functions, and $n(\geq 1), k(\geq 1)$ and $l(\geq 0)$ be three integers such that $\Theta(\infty, f)+$ $\Theta(\infty, g)>\frac{4}{n}$. Suppose $\left[f^{n}(f-1)\right]^{(k)}$ and $\left[g^{n}(g-1)\right]^{(k)}$ share $(1, l)$. If $l \geq 2$ and $n>3 k+11$ or if $l=1$ and $n>5 k+14$, then $f=g$ or $\left[f^{n}(f-1)\right]^{(k)}\left[g^{n}(g-1)\right]^{(k)}=1$.

In this direction recently Abhijith Banerjee [1] proved the following results first one of which improves Theorem G.

Theorem $\mathbf{H}$. Let $f$ and $g$ be two transcendental meromorphic function and $n(\geq 1), k(\geq 1), l(\geq 0)$ be three integers such that $\Theta(\infty, f)+\Theta(\infty, g)>$ $\frac{4}{n}$. Suppose for two nonzero constants $a$ and $b,\left[f^{n}(a f+b)\right]^{(k)}$ and $\left[g^{n}(a g+\right.$ $b)]^{(k)}$ share $(1, l)$. If $l \geq 2$ and $n \geq 3 k+9$ or if $l=1$ and $n \geq 4 k+10$, or if $l=0$ and $n \geq 9 k+18$, then $f=g$ or $\left[f^{n}(a f+b)\right]^{(k)}\left[g^{n}(a g+b)\right]^{(k)}=1$. The possibility $\left[f^{n}(a f+b)\right]^{(k)}\left[g^{n}(a g+b)\right]^{(k)}=1$ does not occur for $k=1$.

Theorem I. Let $f$ and $g$ be two transcendental entire functions, and let $n(\geq 1), k(\geq 1), l(\geq 0)$ be three integers. Suppose for two nonzero constants a and $b,\left[f^{n}(a f+b)\right]^{(k)}$ and $\left[g^{n}(a g+b)\right]^{(k)}$ share $(1, l)$. If $l \geq 2$ and $n \geq 2 k+6$ or if $l=1$ and $n \geq \frac{5 k}{2}+7$, or if $l=0$ and $n \geq 5 k+12$, then $f=g$.

In 2015, Abhijith Banerjee and Pulak Sahoo [3] obtained the following result.

Theorem J. Let $f$ and $g$ be two non-entire transcendental meromorphic functions and let $n(\geq 1), k(\geq 1), l(\geq 0)$ be three integers such that $\Theta(\infty, f)+$ $\Theta(\infty, g)>\frac{4}{n}$. Suppose for two nonzero constants a and $b,\left[f^{n}(a f+b)\right]^{(k)}-P$ and $\left[g^{n}(a g+b)\right]^{(k)}-P$ share $(0, l)$ where $P(\not \equiv 0)$ is a polynomial. If $l \geq 2$ and $n \geq 3 k+9$ or if $l=1$ and $n \geq 4 k+10$ or if $l=0$ and $n \geq 9 k+18$, then $f=g$.

Theorem K. Let $f$ and $g$ be two transcendental entire functions, and let $n(\geq 1), k(\geq 1), l(\geq 0)$ be three integers. Suppose for two nonzero constants $a$ and $b,\left[f^{n}(a f+b)\right]^{(k)}-P$ and $\left[g^{n}(a g+b)\right]^{(k)}-P$ share $(0, l)$ where $P(\not \equiv 0)$ 
is a polynomial. If $l \geq 2$ and $n \geq 2 k+6$ or if $l=1$ and $n \geq \frac{5 k}{2}+7$ or if $l=0$ and $n \geq 5 k+12$, then $f=g$.

The following questions are inevitable.

Quation 1. What can be said if the sharing value zero is replaced by a small function $a$ in the above Theorems $J$ and $K$ ?

Quation 2. Are the Theorems $J$ and $K$ also true for non-constant entire and meromorphic functions?

In this paper, taking the possible answer of the above questions into background we obtain the following results.

Theorem 1. Let $f$ and $g$ be two non-constant meromorphic functions, let $P(w)=a_{m} w^{m}+a_{m-1} w^{m-1}+\ldots+a_{1} w+a_{0}$, for a positive integer $m$ or $P(w) \equiv c_{0}$ where $a_{0}(\neq 0), a_{1} \ldots a_{m-1}, a_{m}(\neq 0), c_{0}(\neq 0)$ are complex constants. Also we suppose that $\left[f^{n} P(f)\right]^{(k)}$ and $\left[g^{n} P(g)\right]^{(k)}$ share $(a, l)$, and $n(\geq 1), k(\geq 1), l(\geq 0)$ are positive integers. Now

(I) when $P(w)=a_{m} w^{m}+a_{m-1} w^{m-1}+\ldots+a_{1} w+a_{0}$, and one of the following conditions holds:

(a) $l \geq 2$ and $n>3 k+m+8$,

(b) $l=1$ and $n>4 k+\frac{3 m+8}{2}$,

(c) $l=0$ and $n>9 k+4 m+14$,

then one of the following three cases holds:

(I1) $f(z) \equiv \operatorname{tg}(z)$ for a constant $t$ such that $t^{d_{1}}=1$, where $d_{1}=\operatorname{gcd}(n+$ $m, n+m-i, \ldots, n), a_{m-i} \neq 0$ for some $i=0,1,2, \ldots, m$,

(I2) $f$ and $g$ satisfy the algebraic equation $R(f, g) \equiv 0$, where $R\left(w_{1}, w_{2}\right)=$ $w_{1}^{n}\left(a_{m} w_{1}^{m}+a_{m-1} w_{1}^{m-1}+\ldots+a_{1} w_{1}+a_{0}\right)-w_{2}^{n}\left(a_{m} w_{2}^{m}+a_{m-1} w_{2}^{m-1}+\ldots+\right.$ $\left.a_{1} w_{2}+a_{0}\right)$, except for $P(w)=a_{1} w+a_{2}$ and $\Theta(\infty ; f)+\Theta(\infty ; g)>\frac{4}{n}$,

(I3) $\left[f^{n} P(f)\right]^{(k)}\left[g^{n} P(g)\right]^{(k)} \equiv a^{2}$, except for $k=1$,

(II) when $P(w) \equiv c_{0}$, and one of the following conditions holds:

(a) $l \geq 2$ and $n>3 k+8$,

(b) $l=1$ and $n>4 k+9$,

(c) $l=0$ and $n>9 k+14$,

then one of the following two cases holds:

(II1) $f \equiv t g$ for some constant $t$ such that $t^{n}=1$,

(II2) $c_{0}^{2}\left[f^{n}\right]^{(k)}\left[g^{n}\right]^{(k)} \equiv a^{2}$. In particular when $n>2 k$ and $a(z)=d_{2}=$ constant, we get $f(z)=c_{1} e^{c z}, g(z)=c_{2} e^{-c z}$, where $c_{1}, c_{2}$ and $c$ are constants satisfying $(-1)^{k} c_{0}^{2}\left(c_{1} c_{2}\right)^{n}(n c)^{2 k}=d_{2}^{2}$.

Theorem 2. Let $f$ and $g$ be two non-constant entire functions, let $P(w)=a_{m} w^{m}+a_{m-1} w^{m-1}+\ldots+a_{1} w+a_{0}$, for a positive integer $m$ or $P(w) \equiv c_{0}$ where $a_{0}(\neq 0), a_{1} \ldots a_{m-1}, a_{m}(\neq 0), c_{0}(\neq 0)$ are complex con- 
stants. Also we suppose that $\left[f^{n} P(f)\right]^{(k)}$ and $\left[g^{n} P(g)\right]^{(k)}$ share $(a, l)$, and $n(\geq 1), k(\geq 1), l(\geq 0)$ are positive integers. Now

(I) when $P(w)=a_{m} w^{m}+a_{m-1} w^{m-1}+\ldots+a_{1} w+a_{0}$, and one of the following conditions holds:

(a) $l \geq 2$ and $n>2 k+m+5$,

(b) $l=1$ and $n>\frac{5 k}{2}+2 m+5$,

(c) $l=0$ and $n>5 k+4 m+8$,

then the conclusion of Theorem 1 holds:

(II) when $P(w) \equiv c_{0}$, and one of the following conditions holds:

(a) $l \geq 2$ and $n>2 k+5$,

(b) $l=1$ and $n>\frac{5 k}{2}+5$,

(a) $l=0$ and $n>5 k+8$,

then the conclusion of Theorem 1 holds.

Theorem 3. Let $f$ and $g$ be two non-constant meromorphic functions and $a(z)(\not \equiv 0, \infty)$ be a small function of $f$ and $g$, let $P(w)=a_{m} w^{m}+$ $a_{m-1} w^{m-1}+\ldots+a_{1} w+a_{0}$, for a positive integer $m$ or $P(w) \equiv c_{0}$ where $a_{0}(\neq 0), a_{1} \ldots a_{m-1}, a_{m}(\neq 0), c_{0}(\neq 0)$ are complex constants. Also we suppose that $f^{n} P(f) f^{\prime}$ and $g^{n} P(g) g^{\prime}$ share $(a, l)$, and $n(\geq 1), k(\geq 1), l(\geq 0)$ are positive integers and one of the following conditions holds:

(a) $l \geq 2$ and $n>m+10$,

(b) $l=1$ and $n>\frac{3 m}{2}+12$,

(c) $l=0$ and $n>4 m+22$,

then one of the following two cases holds:

(I) $f(z) \equiv t g(z)$ for a constant $t$ such that $t^{d_{3}}=1$, where $d_{3}=\operatorname{gcd}(n+m+$ $1, \ldots, n+m+1-i, \ldots, n+1), a_{m-i} \neq 0$ for some $i=0,1,2, \ldots, m$, (II) $f$ and $g$ satisfy the algebraic equation $R(f, g) \equiv 0$, where $R\left(w_{1}, w_{2}\right)=$ $w_{1}^{n+1}\left(\frac{a_{m} w_{1}^{m}}{n+m+1}+\frac{a_{m-1} w_{1}^{m-1}}{n+m}+\ldots+\frac{a_{0}}{n+1}\right)-w_{2}^{n+1}\left(\frac{a_{m} w_{2}^{m}}{n+m+1}+\frac{a_{m-1} w_{2}^{m-1}}{n+m}+\ldots+\frac{a_{0}}{n+1}\right)$,

Theorem 4. Let $f$ and $g$ be two non-constant entire functions and $a(z)(\not \equiv 0, \infty)$ be a small function of $f$ and $g$, let $P(w)=a_{m} w^{m}+a_{m-1} w^{m-1}+$ $\ldots+a_{1} w+a_{0}$, for a positive integer $m$ or $P(w) \equiv c_{0}$ where $a_{0}(\neq 0), a_{1} \ldots a_{m-1}$, $a_{m}(\neq 0), c_{0}(\neq 0)$ are complex constants. Also we suppose that $f^{n} P(f) f^{\prime}$ and $g^{n} P(g) g^{\prime}$ share $(a, l)$, and $n(\geq 1), k(\geq 1), l(\geq 0)$ are positive integers and one of the following conditions holds:

(a) $l \geq 2$ and $n>m+4$,

(b) $l=1$ and $n>\frac{3 m}{2}+6$,

(c) $l=0$ and $n>4 m+11$,

then the conclusion of Theorem 3 holds. 


\section{Lemmas}

In this section we present some lemmas which will be needed in the sequel. Let $F$ and $G$ be two non-constant meromorphic functions defined in $\mathbb{C}$. We shall denote by $H$ the following function:

$$
H=\left(\frac{F^{\prime \prime}}{F^{\prime}}-\frac{2 F^{\prime}}{F-1}\right)-\left(\frac{G^{\prime \prime}}{G^{\prime}}-\frac{2 G^{\prime}}{G-1}\right) .
$$

Lemma 1 ([16]). Let $f$ be a transcendental meromorphic function, and let $P_{n}(f)$ be a differential polynomial in $f$ of the form

$$
P_{n}(f)=a_{n} f^{n}(z)+a_{n-1} f^{n-1}(z)+\ldots+a_{1} f(z)+a_{0},
$$

where $a_{n}(\neq 0), a_{n-1} \ldots a_{1}, a_{0}$ are complex numbers. Then

$$
T\left(r, P_{n}(f)\right)=n T(r, f)+O(1) .
$$

Lemma 2 ([21]). Let $f$ be a non-constant meromorphic function, and $p, k$ be positive integers. Then

$$
\begin{gathered}
N_{p}\left(r, 0 ; f^{(k)}\right) \leq T\left(r, f^{(k)}\right)-T(r, f)+N_{p+k}(r, 0 ; f)+S(r, f), \\
N_{p}\left(r, 0 ; f^{(k)}\right) \leq k \bar{N}(r, \infty ; f)+N_{p+k}(r, 0 ; f)+S(r, f) .
\end{gathered}
$$

Lemma 3 ([9]). Let $F$ and $G$ be two non-constant meromorphic functions sharing $(1,2)$. Then one of the following cases holds:

(i) $T(r) \leq N_{2}(r, 0 ; F)+N_{2}(r, 0 ; G)+N_{2}(r, \infty ; F)+N_{2}(r, \infty ; G)+S(r)$,

(ii) $F=G$,

(iii) $F G=1$. where $T(r)$ denotes the maximum of $T(r, F)$ and $T(r, G)$ and $S(r)=o\{T(r)\}$ as $r \rightarrow \infty$, possibly outside a set of finite linear measure.

Lemma $4([2])$. Let $F$ and $G$ be two non-constant meromorphic functions sharing $(1,1)$ and $H \not \equiv 0$. Then

$$
\begin{aligned}
T(r, F) \leq & N_{2}(r, 0 ; F)+N_{2}(r, 0 ; G)+N_{2}(r, \infty ; F)+N_{2}(r, \infty ; G) \\
& +\frac{1}{2} \bar{N}(r, 0 ; F)+\frac{1}{2} \bar{N}(r, \infty ; F)+S(r, F)+S(r, G) .
\end{aligned}
$$

Lemma 5 ([2]). Let $F$ and $G$ be two non-constant meromorphic functions sharing $(1,0)$ and $H \not \equiv 0$. Then

$$
\begin{aligned}
T(r, F) \leq & N_{2}(r, 0 ; F)+N_{2}(r, 0 ; G)+N_{2}(r, \infty ; F)+N_{2}(r, \infty ; G) \\
& +2 \bar{N}(r, 0 ; F)+\bar{N}(r, 0 ; G)+2 \bar{N}(r, \infty ; F) \\
& +\bar{N}(r, \infty ; G)+S(r, F)+S(r, G) .
\end{aligned}
$$


Lemma 6 ([4]). Let $f, g$ be two non-constant meromorphic functions, let $n, k$ be two positive integers such that $n>2 k$. Suppose $\left[f^{n}\right]^{(k)}$ and $\left[g^{n}\right]^{(k)}$ share $d_{2} C M$. If $\left[f^{n}\right]^{(k)}\left[g^{n}\right]^{(k)} \equiv d_{2}^{2}$, then $f=c_{1} e^{c z}, g=c_{2} e^{-c z}$, where $c_{1}, c_{2}$ and $c$ are constants such that $(-1)^{k}\left(c_{1} c_{2}\right)^{n}(n c)^{2 k}=d_{2}^{2}$.

Lemma 7 ([18]). If $H \equiv 0$, then $F, G$ share $1 C M$. If further $F, G$ share $\infty$ IM then $F, G$ share $\infty C M$.

Lemma 8. Let $f$ and $g$ be two non-constant meromorphic(entire) functions. Let $P(w)$ be defined as in Theorem 1 and $k, m, n>3 k+m(>2 k+m)$ be three positive integers. If $\left[f^{n} P(f)\right]^{(k)} \equiv\left[g^{n} P(g)\right]^{(k)}$, then $f^{n} P(f) \equiv$ $g^{n} P(g)$.

Proof. By the assumption $\left[f^{n} P(f)\right]^{(k)} \equiv\left[g^{n} P(g)\right]^{k}$.

When $k \geq 2$, integrating we get

$$
\left[f^{n} P(f)\right]^{(k-1)} \equiv\left[g^{n} P(g)\right]^{(k-1)}+C_{k-1} .
$$

If possible we suppose $C_{k-1} \neq 0$.

Now in the view of the Lemma 2 for $p=1$ and using the second fundamental theorem we get

$$
\begin{aligned}
(n+m) T(r, f) \leq & T\left(r,\left[f^{n} P(f)\right]^{(k-1)}\right)-\bar{N}\left(r, 0 ;\left[f^{n} P(f)\right]^{(k-1)}\right) \\
& +N_{k}\left(r, 0 ; f^{n} P(f)\right)+S(r, f) \\
\leq & \bar{N}\left(r, 0 ;\left[f^{n} P(f)\right]^{(k-1)}\right)+\bar{N}(r, \infty ; f) \\
& +\bar{N}\left(r, C_{k-1} ;\left[f^{n} P(f)\right]^{(k-1)}\right) \\
& -\bar{N}\left(r, 0 ;\left[f^{n} P(f)\right]^{(k-1)}\right) \\
& +N_{k}\left(r, 0 ; f^{n} P(f)\right)+S(r, f) \\
\leq & \bar{N}(r, \infty ; f)+\bar{N}\left(r, 0 ;\left[g^{n} P(g)\right]^{(k-1)}\right)+k \bar{N}(r, 0 ; f) \\
& +N(r, 0 ; P(f))+S(r, f) \\
\leq & (k+m+1) T(r, f)+(k-1) \bar{N}(r, \infty ; g) \\
& +N_{k}\left(r, 0 ; g^{n} P(g)\right)+S(r, f) \\
\leq & (k+m+1) T(r, f)+(k-1) \bar{N}(r, \infty ; g) \\
& +k \bar{N}(r, 0 ; g)+N(r, 0 ; P(g))+S(r, f) \\
\leq & (k+m+1) T(r, f)+(2 k+m-1) T(r, g) \\
& +S(r, f)+S(r, g) \\
\leq & (3 k+2 m) T(r)+S(r) .
\end{aligned}
$$

Similarly we get

$$
(n+m) T(r, g) \leq(3 k+2 m) T(r)+S(r)
$$


Where $T(r)=\max \{T(r, f), T(r, g)\}$ and $S(r)=\max \{S(r, f), S(r, g)\}$. Combining these we get

$$
(n-m-3 k) T(r) \leq S(r) .
$$

Which is a contradiction since $n>3 k+m$.

Therefore $C_{k-1}=0$ and so $\left[f^{n} P(f)\right]^{(k-1)} \equiv\left[g^{n} P(g)\right]^{(k-1)}$, Repeating $k-1$ times, we obtain

$$
f^{n} P(f)=g^{n} P(g)+c_{0} .
$$

If $k=1$, clearly integrating one we obtain the above. If possible suppose $c_{0} \neq 0$.

Now using the second fundamental theorem we get

$$
\begin{aligned}
(n+m) T(r, f) \leq & \bar{N}\left(r, 0 ; f^{n} P(f)\right)+\bar{N}\left(r, \infty ; f^{n} P(f)\right) \\
& +\bar{N}\left(r, c_{0} ; f^{n} P(f)\right)+S(r, f) \\
\leq & \bar{N}(r, 0 ; f)+m T(r, f)+\bar{N}(r, \infty ; f) \\
& +\bar{N}\left(r, 0 ; g^{n} P(g)\right)+S(r, f) \\
\leq & (m+2) T(r, f)+\bar{N}(r, 0 ; g)+m T(r, g) \\
& +S(r, f)+S(r, g) \\
\leq & (m+2) T(r, f)+(m+1) T(r, g)+S(r, f)+S(r, g) \\
\leq & (2 m+3) T(r)+S(r) .
\end{aligned}
$$

similarly we get

$$
(n+m) T(r, g) \leq(2 m+3) T(r)+S(r)
$$

combining these we get

$$
(n-m-3) T(r) \leq S(r)
$$

which is a contradiction, since $n>m+3$. Therefore $c_{0}=1$ and so

$$
f^{n} P(f) \equiv g^{n} P(g)
$$

This completes the lemma.

Lemma 9. Let $f, g$ be two nonconstant meromorphic (entire functions) and $F=\frac{\left[f^{n} P(f)\right]^{(k)}}{a(z)}, G=\frac{\left[g^{n} P(g)\right]^{(k)}}{a(z)}, n(\geq 1), k(\geq 1), m(\geq 0)$ are positive integers such that $n>3 k+m+3(>2 k+m+2)$ and $P(w)$ be defined as in Theorem 1. If $H \equiv 0$ then

(I) when $P(w)=a_{m} w^{m}+a_{m-1} w^{m-1}+\ldots+a_{1} w+a_{0}$, one of the following three cases holds: 
(I1) $f \equiv t g$ for a constant $t$ such that $t^{d_{1}}=1$, where $d_{1}=\operatorname{gcd}(n+$ $m, \ldots, n+m-i, \ldots, n), a_{m-i} \neq 0$ for some $i=1,2, \ldots, m$,

(I2) $f$ and $g$ satisfy the algebraic equation $R(f, g) \equiv 0$, where $R\left(w_{1}, w_{2}\right)=$ $w_{1}^{n}\left(a_{m} w_{1}^{m}+a_{m-1} w_{1}^{m-1}+\ldots+a_{0}\right)-w_{2}^{n}\left(a_{m} w_{2}^{m}+a_{m-1} w_{2}^{m-1}+\ldots+a_{0}\right)$, except for $P(w)=a_{1} w+a_{2}$ and $\Theta(\infty ; f)+\Theta(\infty ; g)>\frac{4}{n}$,

(I3) $\left[f^{n} P(f)\right]^{(k)}\left[g^{n} P(g)\right]^{(k)} \equiv a^{2}$, (II) when $P(w) \equiv c_{0}$, one of the following two case holds:

(II1) $f \equiv t g$ for some constant $t$ such that $t^{n}=1$,

(II2) $c_{0}^{2}\left[f^{n}\right]^{(k)}\left[g^{n}\right]^{(k)} \equiv a^{2}$. In particular, when $n>2 k$ and $a(z)=d_{2}$ we get $f(z)=c_{1} e^{c z}$ and $g(z)=c_{2} e^{-c z}$, where $c_{1}, c_{2}$ and $c$ are constants satisfying $(-1)^{k} c_{0}^{2}\left(c_{1} c_{2}\right)^{n}(n c)^{2 k}=d_{2}^{2}$.

Proof. Since $H \equiv 0$, by Lemma 7 , we get $F$ and $G$ share 1 CM. On integration we get,

$$
\frac{1}{F-1} \equiv \frac{b G+a-b}{G-1}
$$

where $a, b$ are constants and $a \neq 0$. We now consider the following cases.

Case 1. Let $b \neq 0$ and $a \neq b$. If $b=-1$, then from (3) we have

$$
F \equiv \frac{-a}{G-a-1}
$$

Therefore

$$
\bar{N}(r, a+1 ; G)=\bar{N}(r, \infty ; F)=\bar{N}(r, \infty ; f) .
$$

So in view of Lemma 2 and the second fundamental theorem we get

$$
\begin{aligned}
(n+m) T(r, g) \leq & T(r, G)+N_{k+1}\left(r, 0 ; g^{n} P(g)\right)-\bar{N}(r, 0 ; G) \\
\leq & \bar{N}(r, \infty ; G)+\bar{N}(r, 0 ; G)+\bar{N}(r, a+1 ; G) \\
& +N_{k+1}\left(r, 0 ; g^{n} P(g)\right)-\bar{N}(r, 0 ; G)+S(r, g) \\
\leq & \bar{N}(r, \infty ; g)+N_{k+1}\left(r, 0 ; g^{n} P(g)\right)+\bar{N}(r, \infty ; f)+S(r, g) \\
\leq & \bar{N}(r, \infty ; f)+\bar{N}(r, \infty ; g) \\
& +N_{k+1}\left(r, 0 ; g^{n}\right)+N_{k+1}(r, 0 ; P(g))+S(r, g) \\
\leq & \bar{N}(r, \infty ; f)+\bar{N}(r, \infty ; g)+(k+1) \bar{N}(r, 0 ; g) \\
& +T(r, P(g))+S(r, g) \\
\leq & T(r, f)+(k+m+2) T(r, g)+S(r, f)+S(r, g)
\end{aligned}
$$

without loss of generality, we suppose that there exists a set $I$ with infinite measure such that $T(r, f) \leq T(r, g)$ for $r \in I$.

So for $r \in I$ we have

$$
(n-k-3) T(r, g) \leq S(r, g)
$$


which is a contradiction since $n>k+3$.

If $b \neq-1$, from (3) we obtain that

$$
F-\left(1+\frac{1}{b}\right) \equiv \frac{-a}{b^{2}\left[G+\frac{a-b}{b}\right]} .
$$

So,

$$
\bar{N}\left(r, \frac{(b-a)}{b} ; G\right)=\bar{N}(r, \infty ; F)=\bar{N}(r, \infty ; f) .
$$

Using Lemma 2 and the same argument as used in the case when $b=-1$ we can get a contradiction.

Case 2. Let $b \neq 0$ and $a=b$. If $b=-1$, then from (3) we have

$$
F G \equiv 1 \text {, }
$$

i.e.,

$$
\left[f^{n} P(f)\right]^{(k)}\left[g^{n} P(g)\right]^{(k)} \equiv a^{2}(z),
$$

where $\left[f^{n} P(f)\right]^{(k)}$ and $\left[g^{n} P(g)\right]^{(k)}$ share $a(z) \mathrm{CM}$.

Note that if $P(w) \equiv c_{0}$ then we have

$$
c_{0}^{2}\left[f^{n}\right]^{(k)}\left[g^{n}\right]^{(k)} \equiv a^{2}(z) .
$$

In particular when $n>2 k$ and $a(z)=d_{2}$ then we get by Lemma 6 that $f(z)=c_{1} e^{c z}$ and $g(z)=c_{2} e^{-c z}$, where $c_{1}, c_{2}$ and $c$ are constants satisfying $(-1)^{k} c_{0}^{2}\left(c_{1} c_{2}\right)^{n}(n c)^{2 k}=d_{2}^{2}$.

If $b=-1$, from (3) we have

$$
\frac{1}{F} \equiv \frac{b G}{(1+b) G-1} .
$$

Therefore,

$$
\bar{N}\left(r, \frac{1}{1+b} ; G\right)=\bar{N}(r, 0 ; F) .
$$

So in view of Lemma 2 and the second fundamental theorem we get

$$
\begin{aligned}
(n+m) T(r, g) \leq & T(r, G)+N_{k+1}\left(r, 0 ; g^{n} P(g)\right)-\bar{N}(r, 0 ; G)+S(r, g) \\
\leq & \bar{N}(r, \infty ; G)+\bar{N}(r, 0 ; G)+\bar{N}\left(r, \frac{1}{a+b} ; G\right) \\
& +N_{k+1}\left(r, 0 ; g^{n} P(g)\right)-\bar{N}(r, 0 ; G)+S(r, g) \\
\leq & \bar{N}(r, \infty ; g)+(k+1) \bar{N}(r, 0 ; g)+T(r, P(g)) \\
& +\bar{N}(r, 0 ; F)+S(r, g) \\
\leq & \bar{N}(r, \infty ; g)+(k+1) \bar{N}(r, 0 ; g)+T(r, P(g)) \\
& +(k+1) \bar{N}(r, 0 ; f)+T(r, P(f))+k \bar{N}(r, \infty ; f) \\
& +S(r, f)+S(r, g) \\
\leq & (k+m+2) T(r, g)+(2 k+m+1) T(r, f) \\
& +S(r, f)+S(r, g) .
\end{aligned}
$$


So for $r \in I$ we have

$$
(n-3 k-3-m) T(r, g) \leq S(r, g) .
$$

Which is a contradiction, since $n>3 k+m+3$.

Case 3. Let $b=0$. From (3) we obtain

$$
F \equiv \frac{G+a-1}{a} .
$$

If $a \neq 1$ then from (4) we obtain

$$
\bar{N}(r, 1-a ; G)=\bar{N}(r, 0 ; F) .
$$

We can similarly deduce a contradiction as in case 2 . Therefore $a=1$ and from (4) we obtain

$$
F \equiv G
$$

i.e.,

$$
\left[f^{n} P(f)\right]^{(k)} \equiv\left[g^{n} P(g)\right]^{(k)} .
$$

Note that $n>3 k+m+3>3 k+m$.

So by Lemma 8 , we have

$$
f^{n} P(f) \equiv g^{n} P(g) .
$$

Let $h=\frac{f}{g}$. If $h$ is a constant, putting $f=g h$ in (5) we get

$$
a_{m} g^{n+m}\left(h^{n+m}-1\right)+a_{m-1} g^{n+m-1}\left(h^{n+m-1}-1\right)+\ldots+a_{0} g^{n}\left(h^{n}-1\right)=0,
$$

which implies $h^{d_{1}}=1$, where $d_{1}=\operatorname{gcd}(n+m, \ldots, n+m-i, \ldots, n+1, n)$, $a_{m-i} \neq 0$ for some $i=0,1, \ldots, m$. Thus $f=t g$ for a constant $t$ such that $t^{d_{1}}=1$. where $d_{1}=\operatorname{gcd}(n+m, \ldots, n+m-i, \ldots, n+1, n), a_{m-i} \neq 0$ for some $i=0,1, \ldots, m$.

If $h$ is not a constant, then from (5) we can say that $f$ and $g$ satisfy the algebraic equation $R(f, g)=0$, where

$R\left(w_{1}, w_{2}\right)=w_{1}^{n}\left(a_{m} w_{1}^{n}+a_{m-1} w_{1}^{m-1}+\ldots+a_{0}\right)-w_{2}^{n}\left(a_{m} w_{2}^{n}+a_{m-1} w_{2}^{m-1}+\ldots+a_{0}\right)$.

In particular when $P(w)=a_{1} w+a_{2}$ and $\Theta(\infty, f)+\Theta(\infty, g)>\frac{4}{n}$ then $f \equiv g$. Note that when $P(w) \equiv c_{0}$ then we must have $f \equiv t g$ for some constant $t$ such that $t^{n}=1$.

Lemma 10. Let $f$ and $g$ be two non constant meromorphic functions and $a(z)(\neq 0, \infty)$ be a small function of $f$ and $g$. Let $n$ and $m$ be two positive integers such that $n>\frac{4 m}{t}-(m-1), t$ denotes the number of distinct roots of the equation $P(w) \equiv 0$, where $P(w)$ is defined as in Theorem 3. Then

$$
f^{n} P(f) f^{\prime} g^{n} P(g) g^{\prime} \not \equiv a^{2} .
$$


Proof. First suppose that

$$
f^{n} P(f) f^{\prime} g^{n} P(g) g^{\prime} \equiv a^{2}(z) .
$$

Let $d_{1}$ be the distinct zeros of $P(w)=0$ and multiplicity $P_{i}$, where $i=$ $1,2, \ldots, t, 1 \leq t \leq m$ and $\sum_{i=1}^{t} p_{i}=m$.

Now by the second fundamental theorem for $f$ and $g$ we get respectively

$$
\begin{aligned}
t T(r, f) \leq & \bar{N}(r, 0 ; f)+\bar{N}(r, \infty ; f) \\
& +\sum_{i=1}^{t} \bar{N}\left(r, d_{i} ; f\right)-\overline{N_{0}}\left(r, 0 ; f^{\prime}\right)+S(r, f)
\end{aligned}
$$

and

$$
\begin{aligned}
t T(r, g) \leq & \bar{N}(r, 0 ; g)+\bar{N}(r, \infty ; g) \\
& +\sum_{i=1}^{t} \bar{N}\left(r, d_{i} ; g\right)-\overline{N_{0}}\left(r, 0 ; g^{\prime}\right)+S(r, g)
\end{aligned}
$$

where $\bar{N}_{0}\left(r, 0 ; f^{\prime}\right)$ denotes the reduced counting function of those zeros of $f^{\prime}$ which are not the zeros of $f$ and $f-d_{i}, i=1,2, \ldots, t$ and $\bar{N}_{0}\left(r, 0 ; g^{\prime}\right)$ can be similarly defined.

Let $z_{0}$ be a zero of $f$ with multiplicity $p$ but $a\left(z_{0}\right) \neq 0, \infty$. Clearly $z_{0}$ must be a pole of $g$ with multiplicity $q$. Then from (6) we get $n p+p-1=$ $n q+m q+q+1$. This gives

$$
m q+2=(n+1)(p-q)
$$

From (9) we get $p-q \geq 1$ and so $q \geq \frac{n-1}{m}$. Now $n p+p-1=n q+m q+q+1$ gives $p \geq \frac{n+m-1}{m}$. Thus we have

$$
\bar{N}(r, 0 ; f) \leq \frac{m}{n+m-1} N(r, 0 ; f) \leq \frac{m}{n+m-1} T(r, f) .
$$

Let $z_{1}\left(a\left(z_{1}\right) \neq 0, \infty\right)$ be a zero of $f-d_{i}$ with multiplicity $q_{i}, i=1,2, \ldots t$. Obviously $z_{1}$ must be a pole of $g$ with multiplicity $r(\geq 1)$. Then from $(6)$ we get $p_{i} q_{i}+q_{i}-1=(n+m+1) r+1 \leq n+m+2$. This gives $q_{i} \geq \frac{n+m+2}{p_{i}+1}$ for $i=1,2, \ldots, t$ and so we get

$$
\bar{N}\left(r, d_{i} ; f\right) \leq \frac{p_{i}+1}{n+m+3} N\left(r, d_{i} ; f\right) \leq \frac{p_{i}+1}{n+m+3} T(r, f) .
$$

Clearly

$$
\sum_{i=1}^{d} \bar{N}\left(r, d_{i} ; f\right) \leq \frac{m+t}{n+m+3} T(r, f) .
$$


Similarly we have

$$
\bar{N}(r, 0 ; g) \leq \frac{m}{n+m-1} T(r, g)
$$

and

$$
\sum_{i=1}^{t} \bar{N}\left(r, d_{i} ; g\right) \leq \frac{m+t}{n+m+3} T(r, g) .
$$

Also it is clear that

$$
\text { (14) } \begin{aligned}
\bar{N}(r, \infty ; f) \leq & \bar{N}(r, 0 ; g)+\sum_{i=1}^{t} \bar{N}\left(r, d_{i} ; g\right) \\
& +\bar{N}_{0}\left(r, 0 ; g^{\prime}\right)+S(r, f)+S(r, g) \\
\leq & \left(\frac{m}{n+m-1}+\frac{m+t}{n+m+3}\right) T(r, g)+\bar{N}_{0}\left(r, 0 ; g^{\prime}\right) \\
& +S(r, f)+S(r, g)
\end{aligned}
$$

then by (7), (10), (11) and (14) we get

$$
\begin{aligned}
t T(r, f) \leq & \left(\frac{m}{n+m-1}+\frac{m+t}{n+m+3}\right)\{T(r, f)+T(r, g)\} \\
& +\bar{N}_{0}\left(r, 0 ; g^{\prime}\right)-\bar{N}_{0}\left(r, 0 ; f^{\prime}\right)+S(r, f)+S(r, g) .
\end{aligned}
$$

Similarly we have

$$
\begin{aligned}
t T(r, g) \leq & \left(\frac{m}{n+m-1}+\frac{m+t}{n+m+3}\right)\{T(r, f)+T(r, g)\} \\
& +\bar{N}_{0}\left(r, 0 ; f^{\prime}\right)-\bar{N}_{0}\left(r, 0 ; g^{\prime}\right)+S(r, f)+S(r, g) .
\end{aligned}
$$

Then from (15) and (16) we get

$$
\begin{aligned}
t\{T(r, f)+T(r, g)\} \leq & 2\left(\frac{m}{n+m-1}+\frac{m+t}{n+m+3}\right)\{T(r, f)+T(r, g)\} \\
& +S(r, f)+S(r, g)
\end{aligned}
$$

i.e.,

(17) $\left(t-\frac{2 m}{n+m-1}-\frac{2(m+t)}{n+m+3}\right)\{T(r, f)+T(r, g)\} \leq S(r, f)+S(r, g)$.

Since

$$
\begin{aligned}
& \left(t-\frac{2 m}{n+m-1}-\frac{2(m+t)}{n+m+3}\right) \\
& \quad=\frac{(n+m-1)^{2} t+2(n+m-1)(t-2 m)-8 m}{(n+m-1)(n+m+3)} .
\end{aligned}
$$


We note that when $n+m-1>\frac{4 m}{t}$ i.e., when $n>\frac{4 m}{t}-(m-1)$, then clearly $t-\frac{2 m}{n+m-1}-\frac{2(m+t)}{n+m+3}>0$ and so (17) leads to a contradiction. This completes the proof.

Lemma 11. Let $f, g$ be two nonconstant meromorphic functions, let $F=\frac{f^{n} P(f) f^{\prime}}{a}, G=\frac{g^{n} P(f) g^{\prime}}{a}$, where $P(w)$ is defined as in Theorem 3, $a=$ $a(z)(\not \equiv 0, \infty)$ is a small function with respect to $f$ and $g$, and $n$ is a positive integer such that $n>m+5$. If $H \equiv 0$ then one of the following three cases holds:

(I) $f^{n} P(f) f^{\prime} g^{n} P(g) g^{\prime} \equiv a^{2}(z)$,

(II) $f(z) \equiv t g(z)$ for a constant $t$ such that $t^{d_{3}}=1$, where $d_{3}=\operatorname{gcd}(n+$ $m+1, \ldots, n+m+1-i, \ldots, n+1), a_{m-i} \neq 0$ for some $i=1,2, \ldots, m$,

$(I I I) f$ and $g$ satisfy the algebraic equation $R(f, g) \equiv 0$, where $R\left(w_{1}, w_{2}\right)=$ $w_{1}^{n+1}\left(\frac{a_{m} w_{1}^{m}}{n+m+1}+\frac{a_{m-1} w_{1}^{m-1}}{n+m}+\ldots+\frac{a_{0}}{n+1}\right)-w_{2}^{n+1}\left(\frac{a_{m} w_{2}^{m}}{n+m+1}+\frac{a_{m-1} w_{2}^{m-1}}{n+m}+\ldots+\frac{a_{0}}{n+1}\right)$.

Proof. Clearly

$$
F=\frac{\left[f^{n+1}\left\{\frac{a_{m}}{n+m+1} f^{m}+\frac{a_{m-1}}{n+m} f^{m-1}+\ldots+\frac{a_{0}}{n+1}\right\}\right]^{\prime}}{a}
$$

and

$$
G=\frac{\left[g^{n+1}\left\{\frac{a_{m}}{n+m+1} g^{m}+\frac{a_{m-1}}{n+m} g^{m-1}+\ldots+\frac{a_{0}}{n+1}\right\}\right]^{\prime}}{a},
$$

where

$$
P_{1}(w)=\left\{\frac{a_{m}}{n+m+1} w^{m}+\frac{a_{m-1}}{n+m} w^{m-1}+\ldots+\frac{a_{0}}{n+1}\right\}
$$

proceeding in the same way as the proof of Lemma 9 , taking $k=1$ and considering $n+1$ instead of $n$ we get either

$$
f^{n} P(f) f^{\prime} g^{n} P(g) g^{\prime} \equiv a^{2}(z)
$$

or

$$
f^{n} P(f) f^{\prime} \equiv g^{n} P(g) g^{\prime} .
$$

Let $h=\frac{f}{g}$. If $h$ is a constant, by putting $f=g h$ in the above equation we get

$$
\begin{aligned}
a_{m} g^{m}\left(h^{n+m+1}-1\right)+a_{m-1} g^{m-1}\left(h^{n+m}-1\right)+\ldots \\
+a_{1} g\left(h^{n+2}-1\right)+a_{0}\left(h^{n+1}-1\right) \equiv 0,
\end{aligned}
$$

which implies that $h^{d_{3}}=1$, where $d_{3}=\operatorname{gcd}(n+m+1, \ldots, n+m+1-$ $i, \ldots, n+1), a_{m-i} \neq 0$ for some $i \in\{0,1, \ldots, m\}$. Thus $f \equiv t g$ for a constant $t$ such that $t^{d_{3}}=1$, where $d_{3}=\operatorname{gcd}(n+m+1, \ldots, n+m+1-i, \ldots, n+1)$, $a_{m-i} \neq 0$ for some $i \in\{0,1, \ldots, m\}$. 
If $h$ is not constant then $f$ and $g$ satisfy the algebraic equation $R(f, g) \equiv 0$, where $R\left(w_{1}, w_{2}\right)=w_{1}^{n+1}\left(\frac{a_{m} w_{1}^{m}}{n+m+1}+\frac{a_{m-1} w_{1}^{m-1}}{n+m}+\ldots+\frac{a_{0}}{n+1}\right)-w_{2}^{n+1}\left(\frac{a_{m} w_{2}^{m}}{n+m+1}+\right.$ $\left.\frac{a_{m-1} w_{2}^{m-1}}{n+m}+\ldots+\frac{a_{0}}{n+1}\right)$.

\section{Proof of the theorems}

Proof of Theorem 1. Let $F(z)$ and $G(z)$ be given as in Lemma 9. It follows that $F$ and $G$ share $(1, l)$ except for the zeros and poles of $P(z)$. So from (1) we obtain

$$
\begin{aligned}
N_{2}(r, 0 ; F) \leq & N_{2}\left(r, 0 ;\left[f^{n} P(f)\right]^{(k)}\right)+S(r, f) \\
\leq & T\left(r,\left[f^{n} P(f)\right]^{(k)}\right)-(n+m) T(r, f) \\
& +N_{k+2}\left(r, 0 ; f^{n} P(f)\right)+S(r, f) \\
\leq & T(r, F)-(n+m) T(r, f)+N_{k+2}\left(r, 0 ; f^{n} P(f)\right) \\
& +O\{\log r\}+S(r, f) .
\end{aligned}
$$

Again by (2) we have

$$
N_{2}(r, 0 ; G) \leq k \bar{N}(r, \infty ; f)+N_{k+2}\left(r, 0 ; g^{n} P(g)\right)+S(r, g) .
$$

From (18) we get

$$
\begin{aligned}
(n+m) T(r, f) \leq & T(r, F)+N_{k+2}\left(r, 0 ; f^{n} P(f)\right)-N_{2}(r, 0 ; F) \\
& +O\{\log r\}+S(r, f) .
\end{aligned}
$$

Case 1. Let $H \not \equiv 0$.

Subcase 1 . Let $l \geq 2$. Let $(i)$ of Lemma 3 holds. Then using (19) we obtain from (20),

$$
\begin{aligned}
(n+m) T(r, f) \leq & N_{2}(r, 0 ; G)+N_{2}(r, \infty ; F)+N_{2}(r, \infty ; G) \\
& +N_{k+2}\left(r, 0 ; g^{n} P(f)\right)+O\{\log r\} \\
& +S(r, f)+S(r, g) \\
\leq & N_{k+2}\left(r, 0 ; f^{n} P(f)\right)+N_{k+2}\left(r, 0 ; g^{n} P(g)\right) \\
& +2 \bar{N}(r, \infty ; f)+(k+2) \bar{N}(r, \infty ; g)+O\{\log r\} \\
& +S(r, f)+S(r, g) \\
\leq & (k+m+2)\{T(r, f)+T(r, g)\}+2 \bar{N}(r, \infty ; f) \\
& +(k+2) \bar{N}(r, \infty ; g)+O\{\log r\} \\
& +S(r, f)+S(r, g) \\
\leq & {[(k+m+4)-2 \Theta(\infty ; f)+\epsilon] T(r, f) } \\
& +[(2 k+m+4)-(k+2) \Theta(\infty, g)+\epsilon] T(r, g) \\
& +S(r, f)+S(r, g)
\end{aligned}
$$




$$
\begin{aligned}
\leq & {[(3 k+m+8)-2 \Theta(\infty, f)-2 \Theta(\infty, g)} \\
& -k \min \{\Theta(\infty, f), \Theta(\infty, g)\}+2 \epsilon] T(r)+S(r) .
\end{aligned}
$$

In a similar way we can obtain

$$
\begin{aligned}
(n+m) T(r, g) \leq & {[(3 k+m+8)-2 \Theta(\infty, f)-2 \Theta(\infty, g)} \\
& -k \min \{\Theta(\infty, f), \Theta(\infty, g)\}+2 \epsilon] T(r)+S(r)
\end{aligned}
$$

From (21) and (22) we obtain

$$
\begin{aligned}
{[n-3 k-m-} & 8+2 \Theta(\infty, f)+2 \Theta(\infty, g) \\
& +k \min \{\Theta(\infty, f) \Theta(\infty, g)\}-2 \epsilon] T(r) \leq S(r)
\end{aligned}
$$

contradicting with the fact that $n \geq 3 k+m+8$.

Subcase 2. Let $l=1$, using Lemma 4 and (19) we obtain from (20),

$$
\begin{aligned}
& (n+m) T(r, f) \leq N_{2}(r, 0 ; G)+N_{2}(r, \infty ; F)+N_{2}(r, \infty ; G) \\
& +\frac{1}{2} \bar{N}(r, 0 ; F)+\frac{1}{2} \bar{N}(r, \infty ; F) \\
& +N_{k+2}\left(r, 0 ; f^{n} P(f)\right)+O\{\log r\} \\
& +S(r, f)+S(r, g) \\
& \leq N_{k+2}\left(r, 0 ; f^{n} P(f)\right)+N_{k+2}\left(r, 0 ; g^{n} P(g)\right) \\
& +\frac{1}{2} N_{k+1}\left(r, 0 ; f^{n} P(f)\right)+\frac{k+5}{2} \bar{N}(r, \infty ; f) \\
& +(k+2) \bar{N}(r, \infty ; g)+O\{\log r\} \\
& +S(r, f)+S(r, g) \\
& \leq(k+m+2)\{T(r, f)+T(r, g)\} \\
& +\frac{k+m+1}{2} T(r, f)+\frac{k+5}{2} \bar{N}(r, \infty ; f) \\
& +(k+2) \bar{N}(r, \infty ; g)+O\{\log r\} \\
& +S(r, f)+S(r, g) \\
& \leq\left[\left(2 k+\frac{3 m+10}{2}\right)-\left(\frac{k}{2}+3\right) \Theta(\infty, f)\right. \\
& \left.-\frac{1}{2} \Theta(\infty, f)+\epsilon\right] T(r, f)+[(2 k+m+4) \\
& -\left(\frac{k}{2}+2 g \Theta(\infty, g)-\frac{k}{2} \Theta(\infty, f)+\epsilon\right] T(r, g) \\
& +O\{\log r\}+S(r, f)+S(r, g) \\
& \leq\left[4 k+\frac{5 m+18}{2}-\left(\frac{k+5}{2}\right)(\Theta(\infty, f)\right. \\
& +\Theta(\infty, g))+2 \epsilon] T(r)+S(r) \text {. }
\end{aligned}
$$


Similarly

$$
\begin{aligned}
(n+m) T(r, g) \leq\left[4 k+\frac{5 m+18}{2}\right. & -\left(\frac{k+5}{2}\right)(\Theta(\infty, f) \\
& +\Theta(\infty, g))+2 \epsilon] T(r)+S(r)
\end{aligned}
$$

Combining (23) and (24) we obtain

$$
\left[n-4 k-\frac{5 m+18}{2}+m+\frac{k+5}{2}(\Theta(\infty, f)+\Theta(\infty, g))+2 \epsilon\right] T(r) \leq S(r),
$$

contradiction. Since $n \geq 4 k+\frac{3 m+18}{2}$.

Subcase 3. Let $l=0$, using Lemma 5 and (19) we obtain from (20),

$$
\begin{aligned}
(25)(n+m) T(r, f) \leq & N_{2}(r, 0 ; G)+N_{2}(r, \infty ; F)+N_{2}(r, \infty ; G) \\
& +2 \bar{N}(r, 0 ; F)+\bar{N}(r, 0 ; G)+N_{k+2}\left(r, 0 ; f^{n} P(f)\right) \\
& +2 \bar{N}(r, \infty ; F)+\bar{N}(r, \infty ; G) \\
& +O\{\log r\}+S(r, f)+S(r, g) \\
\leq & N_{k+2}\left(r, 0 ; f^{n} P(f)\right)+N_{k+2}\left(r, 0 ; g^{n} P(g)\right) \\
& +2 N_{k+2}\left(r, 0 ; f^{n} P(f)\right)+N_{k+1}\left(r, 0 ; g^{n} P(g)\right) \\
& +(2 k+4) \bar{N}(r, \infty ; f)+(2 k+3) \bar{N}(r, \infty ; g) \\
& +O\{\log r\}+S(r, f)+S(r, g) \\
\leq & {[(5 k+3 m+8)-(2 k+4) \Theta(\infty ; f)-\epsilon] T(r, f) } \\
& +[(4 k+2 m+6)-(2 k+3) \Theta(\infty ; g)-\epsilon] T(r, g) \\
& +O\{\log r\}+S(r, f)+S(r, g) \\
& +[(9 k+5 m+14)-(2 k+3)[\Theta(\infty ; f) \\
& +\Theta(\infty, g)]-\min \{\Theta(\infty, f) \Theta(\infty ; g)\} \\
& +2 \epsilon] T(r)+S(r) .
\end{aligned}
$$

Similarly

(26) $(n+m) T(r, g) \leq[(9 k+5 m+14)-(2 k+3)[\Theta(\infty ; f)+\Theta(\infty, g)]$ $-\min \{\Theta(\infty, f) \Theta(\infty ; g)\}+2 \epsilon] T(r)+S(r)$.

From (25) and (26) we get

$$
\begin{aligned}
{[n-9 k-4 m} & -14]+(2 k+3)(\Theta(\infty, f)+\Theta(\infty ; g)) \\
& +\min \{\Theta(\infty ; f) \Theta(\infty ; g)\}-2 \epsilon] T(r) \leq S(r),
\end{aligned}
$$

contradicts with the facts that $n \geq 9 k+4 m+14$. 
Case 3. Let $H \equiv 0$. Then the Theorem follows from Lemma 9 .

Proof of Theorem 2. Noting that $\bar{N}(r, \infty ; f)=0, \bar{N}(r, \infty ; g)=0$ and proceeding in the like manner as the proof of Theorem 1 we obtain the result of the Theorem 2 .

Proof of Theorem 3. Let $F=\frac{f^{n} P(f) f^{\prime}}{a(z)}$ and $G=\frac{g^{n} P(g) g^{\prime}}{a(z)}$. Then $F, G$ share $(1, l)$, except the zeros and poles of $a(z)$. Clearly

$$
F=\frac{\left[f^{n+1}\left\{\frac{a_{m}}{n+m+1} f^{m}+\frac{a_{m-1}}{n+m} f^{m-1}+\ldots+\frac{a_{0}}{n+1}\right\}\right]^{\prime}}{a}
$$

and

$$
G=\frac{\left[g^{n+1}\left\{\frac{a_{m}}{n+m+1} g^{m}+\frac{a_{m-1}}{n+m} g^{m-1}+\ldots+\frac{a_{0}}{n+1}\right\}\right]^{\prime}}{a},
$$

where

$$
P_{1}(w)=\left\{\frac{a_{m}}{n+m+1} w^{m}+\frac{a_{m-1}}{n+m} w^{m-1}+\ldots+\frac{a_{0}}{n+1}\right\} .
$$

Case 1. Let $H \not \equiv 0$. Now following the same procedure as adopted in the proof of Case 1 of Theorem 1 we can easily deduce a contradiction.

Case 2. Let $H \equiv 0$. Since $n>k_{1}$ and $n>m+5$ the theorem follows from Lemma 10 and 11.

Proof of Theorem 4. Noting that $\bar{N}(r, \infty ; f)=0, \bar{N}(r, \infty ; g)=0$ and proceeding in the like manner as the proof of Theorem 3 we obtain the result of the Theorem 4.

\section{References}

[1] BANERJEE A., Uniqueness of certain non-linear differential polynomials sharing 1-points, Kyungpook Math. J., 51(1)(2011), 43-58.

[2] BAnerJee A., Meromorphic functions sharing one value, Int. J. Math. Math. Sci., 22(2005), 3587-3598.

[3] BanerJee A., Sahoo P., Certain nonlinear differential polynomials sharing a nonzero polynomial with finite weight, Kyungpook Math. J., 55(2015), 653-666.

[4] Banerjee A., Majumder S., Non-linear differential polynomials sharing small function with finite weight, Arab. J. Math. (Springer), 4(1)(2015), 7-28.

[5] FAng M.-L., Uniqueness and value-sharing of entire functions, Comput. Math. Appl., 44(5-6)(2002), 823-831.

[6] FAng C.-Y., FAng M.-L., Uniqueness of meromorphic functions and differential polynomials, Comput. Math. Appl., 44(5-6)(2002), 607-617.

[7] Hayman W.K., Picard values of meromorphic functions and their derivatives, Ann. of Math., 70(2)(1959), 9-42.

[8] Hayman W.K., Meromorphic functions, Oxford Mathematical Monographs, Clarendon Press, Oxford, 1964. 
[9] LAHIRI I., Weighted value sharing and uniqueness of meromorphic functions, Complex Variables Theory Appl., 46(3)2001), 241-253.

[10] LAhiri I., Value distribution of certain differential polynomials, Int. J. Math. Math. Sci., 28(2)(2001), 83-91.

[11] Lahiri I., On a question of Hong Xun Yi, Arch. Math. (Brno), 38(2)(2002), 119-128.

[12] LAhiRi I., Dewan S., Value distribution of the product of a meromorphic function and its derivative, Kodai Math. J., 26(1)(2003), 95-100.

[13] LI J.-D., Uniqueness of meromorphic functions and differential polynomials, Int. J. Math. Math. Sci., (2011), Art. ID 514218, 12 pp.

[14] Lin W., Yi H., Uniqueness theorems for meromorphic functions concerning fixed-points, Complex Var. Theory Appl., 49(11)(2004), 793-806.

[15] XU H.-Y., Yi C.-F., CAO T.-B., Uniqueness of meromorphic functions and differential polynomials sharing one value with finite weight, Ann. Polon. Math., 95(1)(2009), 51-66.

[16] Yang C.C., On deficiencies of differential polynomials II, Math. Z., 125 (1972), 107-112.

[17] YANG L., Value distribution theory, translated and revised from the 1982 Chinese original, Springer, Berlin, 1993.

[18] Yi H.-X., Meromorphic functions that share one or two values II, Kodai Math. J., 22(2)(1999), 264-272.

[19] Yi H.X., YANG C.C., Uniqueness theory of meromorphic functions, Science Press, Beijing, 1995.

[20] Zhang J., Uniqueness theorems for entire functions concerning fixed points, Comput. Math. Appl., 56(12)(2008), 3079-3087.

[21] Zhang J.-L., YAng L.-Z., Some results related to a conjecture of R. Brück, JIPAM. J. Inequal. Pure Appl. Math., 8(1)(2007), Article 18, 11 pp.

\author{
WAGHAMORE P. HARINA \\ Department of Mathematics \\ Central College Campus \\ BANGALORE UNIVERSITY \\ BANGALORE-560 001, INDIA \\ e-mail: harinapw@gmail.com
S. RAJESHWARI
Department of Mathematics
Central College Campus
BANGALORE UNIVERSITY
BANGALORE-560 001, INDIA
e-mail: rajeshwaripreetham@gmail.com

Received on 05.12.2016 and, in revised form, on 23.05.201\%. 